\title{
KEY ISSUES IN A BENCHMARKING EXERCISE FOR LONGITUDINAL TENSILE FAILURE OF UNIDIRECTIONAL COMPOSITES
}

\author{
Christian Breite, Arsen Melnikov, Francisco Mesquita, Stepan V. Lomov, Yentl Swolfs"
}

KU Leuven, Department of Materials Engineering, Kasteelpark Arenberg 44, 3001, Leuven, Belgium *yentl.swolfs@kuleuven.be

Longitudinal tensile failure of unidirectional plies is a key failure mode for laminated composites. We therefore organised a benchmarking exercise with 7 participating models, where the models were carefully compared against each other based on two virtual materials [1]. We also performed a detailed experimental validation study for 6 of the participating models, based on synchrotron computed tomography data for fibre break development combined with carefully and objectively measured input data. The present paper analyses where the discrepancies between models and experiments may have arisen from, based on the KU Leuven strength model [1]. Fig. 1 for example shows how the scatter in the experimental fibre break density evolutions is similar to the scatter in the Monte Carlo simulations. The scatter bands however do not overlap. We also fitted a Weibull distribution to the fibre break density evolution (excluding clusters of fibre breaks). Running simulations with the fitted Weibull distribution as input revealed that a good agreement between the density developments can be achieved. However, even a fitted Weibull distribution still leads to significant errors in other parameters, such as tensile strength or cluster evolution. The complete analysis of all key issues has shown that the discrepancies cannot be attributed to any single input parameter or assumption, such as the Weibull distribution, but should be attributed to a combination of unknowns that need to be explored in further studies.

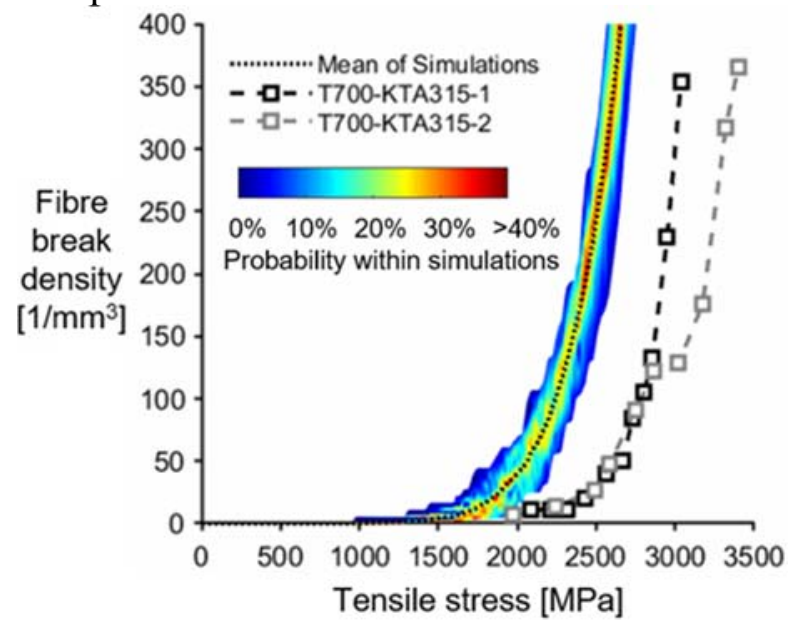

Figure 1. Comparison of the scatter in the fibre break density evolution for the experiments versus for the Monte Carlo simulations.

This benchmark was performed within the framework of the FiBreMoD project and has received funding from the European Union's Horizon 2020 research and innovation programme under the Marie Skłodowska-Curie grant agreement No 722626.

\section{References}

[1] C. Breite, A. Melnikov, A. Turon, A.B. de Morais, F. Otero, F. Mesquita, et al. (2021) Blind benchmarking of seven longitudinal tensile failure models for two virtual unidirectional composites. Composites Science and Technology, 202, 108555.

[2] F. Mesquita, Y. Swolfs, S.V. Lomov, L. Gorbatikh. Ply fragmentation in unidirectional hybrid composites linked to stochastic fibre behaviour: A dual-scale model. Composites Science and Technology, 181, 107702. 